\title{
Francine Couture
}

Sociologue de l'art, Département d'histoire de l'Art, UQAM

1988

\section{"Projet politique, projet artistique"}

Un document produit en version numérique par Jean-Marie Tremblay, bénévole, professeur de sociologie au Cégep de Chicoutimi

Courriel: jmt_sociologue@,videotron.ca Site web: http://www.uqac.ca/jmt-sociologue/

Dans le cadre de la collection: "Les classiques des sciences sociales" Site web: http://www.uqac.ca/Classiques_des_sciences_sociales/

Une collection développée en collaboration avec la Bibliothèque Paul-Émile-Boulet de l'Université du Québec à Chicoutimi

Site web: http://bibliotheque.uqac.ca/ 
Cette édition électronique a été réalisée par Jean-Marie Tremblay, bénévole, professeur de sociologie au Cégep de Chicoutimi à partir de :

Francine Couture

Sociologue de l'art, Département d'histoire de l'Art, UQAM

\section{"Projet politique, projet artistique"}

Un article publié dans l'ouvrage sous la direction de Jean-François Léonard, Georges-Émile Lapalme, pp. 151-157. Montréal: Les Presses de l'Université du Québec, 1988, 303 pp. Collection: Les leaders politiques du Québec contemporain.

[Autorisation accordée le 6 janvier 2005 par Mme Francine Couture.]

81 Courriel: $\quad$ Mme Francine Couture : couture.francine@uqam.ca

sPolices de caractères utilisée :

Pour le texte: Times, 12 points.

Pour les citations : Times 10 points.

Pour les notes de bas de page : Times, 10 points.

Édition électronique réalisée avec le traitement de textes Microsoft Word 2004 pour Macintosh.

Mise en page sur papier format : LETTRE (US letter), 8.5' $x 11$ '')

Édition numérique réalisée le 20 juillet 2005 à Chicoutimi, Ville de Saguenay, province de Québec, Canada.

\section{CFait avec}




\title{
Table des matières
}

\author{
Introduction
}

1. Les convictions personnelles de Georges-Émile Lapalme sur la culture.

2. Borduas

3. La création du ministère des affaires culturelles 
Francine Couture

Sociologue de l'art, Département d'histoire de l'Art, UQAM

\section{"Projet politique, projet artistique"}

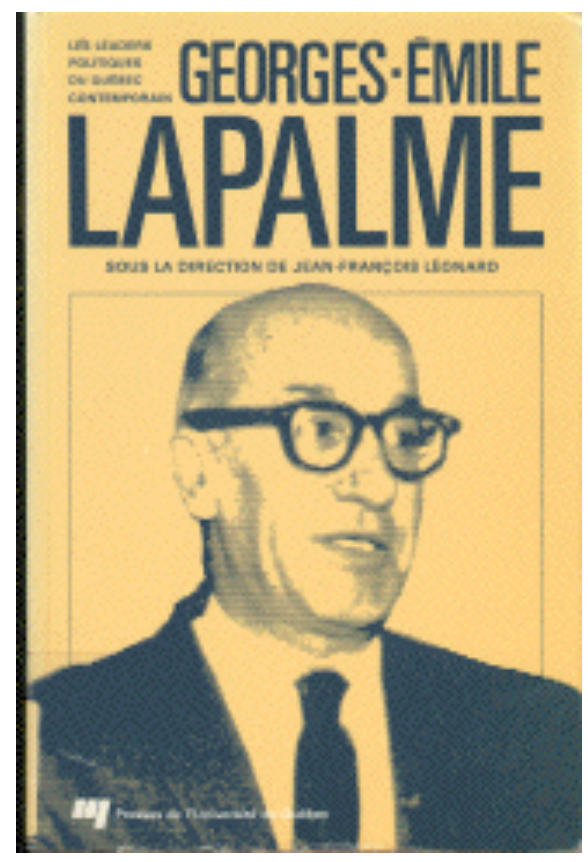

Un article publié dans l'ouvrage sous la direction de Jean-François Léonard, Georges-Émile Lapalme, pp. 151-157. Montréal: Les Presses de l'Université du Québec, 1988, 303 pp. Collection: Les leaders politiques du Québec contemporain. 


\section{Introduction}

En tant qu'historienne d'art intervenant dans un colloque sur l'activité d'un homme politique qui a été ministre des Affaires culturelles, je ne peux que m'interroger sur le phénomène de l'appropriation de la sphère artistique par les hommes politiques. Et je me pose cette interrogation à propos des arts visuels. Une manifestation publique importante de ce phénomène a été la création du ministère des Affaires culturelles au Québec. Georges-Émile Lapalme a été à l'origine de cette création qui avait été une promesse électorale du Parti libéral, en 1960.

J'ai recueilli un certain nombre de données concernant la période qui correspond à la création du ministère des Affaires culturelles jusqu'à la démission de Georges-Émile Lapalme, soit de 1961 à 1964. Je vous fais donc part des observations que j'ai tirées de ces données. 


\section{1. \\ Les convictions personnelles de Georges-Émile Lapalme sur la culture.}

$\underline{\text { Retour à la table des matières }}$

Comme les autres participants à ce colloque, j'ai lu les Mémoires de GeorgesÉmile Lapalme. Ces Mémoires étant de l'ordre du discours privé, ils nous permettent d'évaluer à quel point Lapalme était personnellement convaincu de l'importance fondamentale de l'activité artistique dans une société. Ses convictions personnelles sont devenues les objectifs des premières initiatives de son ministère. On constate aussi qu'il défendait les valeurs de modernité en art, valeurs qui, au début des années 60 dans le cas des arts visuels, s'incarnaient dans l'art abstrait, prolongement des recherches artistiques entreprises par les automatistes au début des années 40. Lapalme s'opposait à une vision de la culture traditionnelle qu'il appelait la culture du terroir. Par exemple, dans ses Mémoires, il déplore qu'en 1953, à son arrivée à Québec, le budget affectée à la collection «peinture », au Musée du Québec, serve à acheter des coqs gaulois et des ciboires ${ }^{1}$. "N'ai-je pas déjà écrit, affirme-t-il, que nous n'avions pas un seul Riopelle ${ }^{2}$."

Cette opinion n'était pas partagée par ses collègues de l'appareil politique, loin d'être convaincus de la valeur culturelle de l'art abstrait. Il faut aussi dire que les préjugés contre ce courant artistique étaient forts présents dans la mentalité de l'époque. On peut encore lire par exemple dans L'Action catholique de décembre 1960 : "l'art abstrait, une affaire bien montée pour les communistes et les gens snobs ${ }^{3 "}$. Les valeurs de modernité, comme le caractère individuel de la production artistique ou la liberté d'expression du sujet créateur, posaient

Ce budget s'élevait à 35000 dollars par année. Georges-Émile Lapalme, Le Paradis $d u$ pouvoir, Mémoires, t. III, Montréal, Leméac, collection Vies et mémoires, p. 157.

Ibid.

L'Action catholique, 9-12-60. 
problème à plusieurs membres du milieu politique d'alors. L'activité artistique ou intellectuelle était encore perçue comme une activité menaçante, subversive, pouvant mettre en danger le consensus social. Cette opinion était celle de opinion était celle de l'Union Nationale, qui a pu l'exprimer notamment au moment de la création du ministère des Affaires culturelles : les journaux de l'époque rapportent que ses partisans craignaient que le ministère devienne le refuge d'“esprits supérieurs qui ne trouvent rien de bon dans nos institutions présentes 4". L'Union Nationale avait également peur que les artistes soient embrigadés par la doctrine politique ou même, advenant la prise du pouvoir politique par un mouvement de gauche, que les artistes et les intellectuels trouvent dans un ministère des Affaires culturelles "un outil pour amener la population à partager sa façon de voir les choses ${ }^{5 "}$. Ces idées étaient aussi partagées par l'équipe du journal L'Action catholique.

Georges-Émile Lapalme devait défier ces courants d'opinions lorsqu'il voulait faire acheter une oeuvre d'art par le Conseil du Trésor. Il faut se rappeler qu'à cette époque où le champ artistique n'était pas fermement consolidé l'appréciation de la valeur culturelle d'une oeuvre d'art appartenait à l'appareil politique ; cette appréciation n'était pas médiatisée par les experts du milieu artistique, mais elle pouvait être le privilège du Conseil du Trésor. Les Mémoires de Lapalme contiennent plusieurs remarques à ce sujet. Lapalme évoque notamment de l'époque où il a voulu faire acheter un Riopelle ("... le seul Riopelle que possédait le Québec en 1964") ${ }^{6}$ pour la collection du gouvernement. L'acquisition de ce Riopelle avait été recommandée par Guy Viau, qui était alors directeur du Musée du Québec. Le Conseil du Trésor envoya un fonctionnaire à la galerie - la Galerie Hébert, galerie qui défendait l'abstraction lyrique ou le post-automatisme - pour évaluer le tableau ${ }^{7}$. Georges-Émile Lapalme s'indigna de l'autorité qu'avait le Conseil du Trésor en matière de culture. Et, comme chacun sait, ce sentiment d'indignation et même d'impuissance à faire reconnaître l'importance de la culture par le Conseil du Trésor entraînera sa démission en 1964.

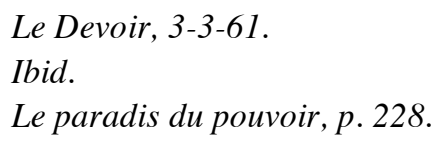

M. Lapalme a dit les résistances qu'a opposées le contrôleur de la trésorerie quand le ministère a décidé d'acquérir un Riopelle pour le Musée de Québec. Ce fonctionnaire des Finances ne cachait pas ses goûts : il avait, on s'en souvient, chargé un de ses agents d'examiner le tableau et, sur le rapport qu'il en avait reçu, empêché l'achat : seule une colère du ministre parvint, in extremis, à lever l'obstacle. Lorsque nous réussîmes à négocier avec la France une grande rétrospective Rouault, le contrôleur avait pris sa retraite définitive. La nouvelle de l'exposition dans. la presse le frappa. Il découpa l'information et l'adressa à son successeur, qui me la fit voir, avec dans les marges des commentaires qui étaient à peu près ceux-ci : "Peinture moderne. Si j'avais été là, je n'aurais pas permis qu'on fasse des dépenses pour montrer ces horreurs" (Guy Frégault, Chronique des années perdues, Montréal, Leméac, 1976, p. 120). 


\section{2.}

\section{Borduas}

\section{Retour à la table des matières}

Dans le champ des arts visuels, un événement important survient en 1960 : la mort de Borduas. Cette mort entraîne une réévaluation de l'importance de son oeuvre dans l'histoire de l'art et une réflexion sur la place qu'occupe l'art dans la société québécoise. Il est pertinent de souligner que la reconnaissance de l'œuvre de Borduas, après sa mort, a été l'apanage d'institutions artistiques extérieures à la communauté francophone. Dès janvier 196 1, par exemple, une rétrospective Borduas est organisée à Amsterdam, au Musée Stedejik ${ }^{8}$; il s'agit de la première rétrospective posthume de l'œuvre de Borduas sur la scène internationale. La rétrospective suivante sera le fait du Musée des Beaux Arts de Montréal, en $1962{ }^{9}$, musée à ce moment-là dirigé par un Américain. Cette exposition fut aussi présentée à la Galerie Nationale du Canada et à l'Art Galery of Toronto. A l'époque, il n'y a pas encore de Musée d'art contemporain à Montréal. Du côté francophone, la valorisation de l'oeuvre de Borduas provient surtout d'individus isolés, c'est-à-dire des artistes et des intellectuels qui collaborent à des revues comme Liberté ${ }^{10}$ et Cité libre : la conscience du public qu'on éveille à ce moment-là, le sens de l'oeuvre mais aussi de la démarche de ce peintre, se résume à Borduas, un modèle à suivre, un modèle d'authenticité, d'exigence intellectuelle, de recherche de liberté ${ }^{11}$. On le présente surtout comme un héros persécuté, comme un martyr qui a donné sa vie pour la révolution culturelle au Québec.

22-12-1960 au 20-1-61 : Rétrospective Borduas à Amsterdam au Stedejik Museum dont le directeur était W.J.H.B. Sandberg.

9 11-1-62 au 11-2-62 : Rétrospective Borduas, 1905-1960, Musée des Beaux-Arts de Montréal, Galerie Nationale du Canada et Art Gallery of Toronto.

10 La revue Liberté, numéros nov.-déc. 1960, mars-avril 1961, janv.-fév. 1962, avril 1962.

11 Voici par exemple ce qu'a écrit Pierre Vadeboncoeur: "Borduas est à la source de la conscience des intellectuels depuis quinze ans chez nous : il est le point de départ et le point central de cette conscience". Et il ajoute : "il ne faudra jamais être faux, Borduas nous a donné ce message. Il avait quelque chose de saint" (Cité libre, janv. 1961, Xle année, no. 33, p. 30.) 
Est mise aussi en valeur la dimension collective de la démarche picturale de Borduas. À ce titre, l'interprétation que Robert Elie fait de l'oeuvre de Borduas est exemplaire. Robert Élie a été directeur de l'École des Beaux-Arts de Montréal, et a permis, à la fin des années 50, que la démarche automatiste guide l'enseignement des arts plastiques à cette école. Premier attaché culturel nommé par Lapalme à la Délégation du Québec à Paris, Élie présente, dans un texte publié en 1960, la recherche de Borduas en peinture comme le symbole de la lutte pour l'émancipation de la société québécoise. Il décrit la démarche du peintre en quelque sorte comme une métaphore de la société québécoise, qui est à une étape de découvertes et de recherches :

Il y aurait bien une autre nuit où il pouvait revenir à la toile inachevée, à la lourde matière qui était notre réalité, à un combat épuisant qui se terminerait une fois sur deux par un échec aussitôt repris, mais une fois sur deux par une victoire comme il s'en est peu remportée dans notre pays. Et l'impossible parlait comme le possible, l'inconnu ne faisait plus peur aux enfants, les frontières étaient abolies. C'était un monde neuf et touffu que l'on commençait à peine à interroger ${ }^{12}$."

Cette métaphore est reprise par G.-É. Lapalme, lorsque, dans une intervention à l'Assemblée législative le 3 mars $1964^{13}$ - au moment de la création du Musée d'art contemporain -, il affirme, parlant de la peinture : "C'est le domaine peut-être le plus important de notre culture à l'heure actuelle, en tant que l'extériorisation de nos talents qui se fait à l'étranger. C'est dans la peinture que nous sommes des maîtres à l'heure actuelle, si nous en sommes." Cette idée-là est partagée par le milieu des artistes et des intellectuels et par Robert Elie. Celui-ci, dans des textes publiés entre 1958 et 1961, nous dira que la société québécoise, animée par l'idée de progrès, ne peut que favoriser le développement d'un art vivant. Ce n'est qu'en intégrant l'art vivant comme valeur sociale que la société pourra réaliser son projet de rupture avec les valeurs et les modes de vie anciens. Pour Robert Élie et pour beaucoup d'autres artistes et intellectuels à l'époque, l'art a une dimension éthique. En 1958, il écrit dans la Revue dominicaine "Sans un art vivant, une société meurt d'ennui ${ }^{14 ",}$ "... C'est la grandeur de notre temps jeté en plein

12 R. Élie, "Rencontre avec Borduas", La Presse, 27-2-1960, p. 33 - réédité dans Oeuvres, La Salle, Québec, Hurtubise, HMH, 1979, p. 602.

13 Débats de l'Assemblée législative du Québec, le mardi 3 mars 1964, vol. 1, no. 34, p. 1431.

14 R. Élie, "Les arts et la société", Revue dominicaine, vol. LXIV, t. III, nov. 1958, pp. 214-219, réédité dans Oeuvres, p. 686 : "Si la société ne favorise pas ces explorations en espace libre, elle n'est plus qu'une prison où l'homme a le choix entre mourir d'inanition et étouffer la rage."

Et tout en défendant la nécessité de la présence d'un art de recherche dans la société, Robert Élie défend les principes de liberté absolue du sujet créateur. Voici ce qu'il écrit : "En un sens l'art ne saurait s'intégrer à la société, i.e., être asservi à ses fins puisqu'il s'épanouit audelà des frontières du social. Mais la destinée de l'homme ne peut s'accomplir sans accéder à cet au-delà, il faut donc que la société qu'il constitue fasse place à l'art et d'autres activités dites inutiles mais sans prétendre à aucun droit de contrôle ou de censure." 
inconnu et il nous faut accepter tous les risques de l'aventure si nous voulons vivre et si nous voulons produire des oeuvres vivantes ${ }^{15}$. Robert Élie, dans une conférence devant la Chambre de Commerce ${ }^{16}$ dira aussi : "Se réfugier dans la contemplation des chefs d'œuvres du passé c'est encore refuser de vivre."

G.-É. Lapalme aussi évoque le sort fait à Borduas ${ }^{17}$ : "J'avais toujours considéré comme pitoyable la place que l'État avait réservée aux artistes, particulièrement aux peintres. Le sort fait à Borduas m'avait paru inique ${ }^{18}$." Il a voulu corriger cette situation, sortir l'artiste de la marginalité. Il considérait que l'État devait accorder à l'artiste une place dans la société et améliorer sa qualité de vie.

\section{3. \\ La création du ministère des affaires culturelles}

$\underline{\text { Retour à la table des matières }}$

La création du ministère des Affaires culturelles suppose la reconnaissance de la dimension collective de l'art; qu'on reconnaisse en somme que l'activité artistique s'inscrit dans une communauté, qu'elle joue un rôle social, qu'elle est un définisseur important de la société québécoise qui, au début des années 60 , affirme sa modernité. L'intervention de l'État dans la vie artistique implique aussi que l'État se reconnaisse une nouvelle responsabilité soit celle d'aider les artistes à réaliser la dimension collective de leur engagement ${ }^{19}$. En 1962, par exemple,

lbid., p. 688.

lbid., 29-11-60, p. 747.

On trouve dans les journaux la mention d'un projet de Musée Borduas à partir de la maison du peintre Ozias Leduc ou de celle de Borduas (Le Nouveau Journal, 17-1-62).

18 G.-É. Lapalme, Le Paradis du pouvoir, p. 157.

19 "Je professerai bientôt de croire, et je le croirai en effet, que l'État du Québec est entré dans une ère nouvelle le jour où il a mis sur pied le ministère. De ce jour, affirmerais-je, les services destinés à favoriser les travaux de l'esprit ont cessé d'être des objets de luxe déposés dans les vitrines d'administrations plus coquettes que d'autres et ont accédé ainsi au nombre des réalités dont la collectivité se préoccupe d'une façon normale et quotidienne, en y mettant 
Georges-Émile Lapalme prononce une conférence au Symposium sur le Canada français à l'Université de Colombie-britannique où il exprime sa vision du Canada français moderne : une société dynamique, dit-il, qui prend en main son destin national et international et de son nouveau pouvoir sur le monde. Et il donne en exemple le travail de jeunes écrivains comme Jacques Godbout et Yves Préfontaine qui, dit-il, participent à ce processus de modernisation de la société québécoise.

Parlons maintenant des réalisations. On sait que les premiers moments des Affaires culturelles ont été pénibles : le gouvernement du Québec n'a pas pu jouer totalement son rôle de mécène. Certaines choses ont quand même été faites, et ont permis la création d'un espace social pour l'art. Je pense, entre autres, à la mise sur pied de programmes d'aide à la création ${ }^{20}$. Le musée du Québec ${ }^{21}$, qui était alors un musée aux multiples fonctions, est devenu exclusivement un musée d'art. Il ne semble pas qu'au début des années 60 il y ait eu une volonté politique explicitement exprimée de créer un musée d'art contemporain; le projet gouvernemental de musée concernait exclusivement le Musée du Québec qui était déjà un musée d'État. L'initiative de la création d'un musée d'art contemporain provient de la communauté artistique, soit d'un groupe de peintres réunis autour du propriétaire de la Galerie Soixante, Otto Bengle, qui demanda aux artistes de donner chacun une toile pour constituer la première collection du musée ${ }^{22}$. Depuis les années 40, des groupes d'artistes appuyés par des critique d'art, marchands de tableaux et collectionneurs avaient réussi à développer un espace social spécifique de l'art. Il manquait un musée pour montrer la continuité de l'art contemporain québécois; propriétaires de galeries, artistes et critiques d'art qui défendaient les valeurs de l'art contemporain, au début des années 60 , réclamaient

le prix, et non plus seulement les jours de fête et à l'heure des discours, en y mettant surtout de la bonne volonté. J'ai eu la foi" (Guy Frégault, op. cit., p. 19.)

201961 : Dans un mémoire déposé au ministère des Affaires culturelles Y. Lasnier, L. Belzile, U. Comtois, L. Gervais, J. Goguen, R. Letendre, J. McEwen, J.-P. Mousseau, C. Vermette, F. Toupin, tous ces artistes demandent à l'État la création de programmes de bourses, d'expositions, de déductions d'impôt, de construction d'ateliers, d'intégration des arts à l'architecture (Le Devoir, 18-3-61).

En 1962 le ministère des Affaires culturelles a redéfini les règlements du Concours artistique de la province et a augmenté son budget.

21 Le 21 avril 1962 est créée une Commission consultative sur l'orientation du Musée du Québec, dont les membres sont le directeur du Musée, Gérard Morisset, Claude Beaulieu, directeur de la revue Vie des Arts, et le peintre Jean-Paul Lemieux (Guy Frégault, op. cit., pp. 23-24).

22 Ce groupe était aussi appuyé par d'anciens directeurs de l'École des Beaux-Arts de Montréal, Robert Elie et Edmond Labelle, ainsi que par Jean-René Ostiguy, Guy Viau et Jean Simard qui étaient membres du Conseil provincial des arts (Mémoires de l'Association des arts plastiques, le 12 mars 1965 ; président: G. Molinari; comité du mémoire: Claude Tousignant, Yves Gaucher, Gilbert Maison ; rédacteur et aviseur technique : Yves Robillard). 
solidairement un tel musée. Georges-Émile Lapalme a soutenu ce groupe et a obtenu du gouvernement la somme de 100000 dollars pour créer un Musée d'art contemporain, qui fut inauguré par l'exposition du peintre français Rouault.

Pourquoi inaugurer un musée d'art contemporain québécois avec l'œuvre d'un peintre français ? En fait, l'exposition Rouault ${ }^{23}$ avait été pilotée par le sousministre des Affaires culturelles, Guy Frégault, et son attaché culturel à Paris, Robert Élie. Cette exposition répondait aussi à une volonté de développer des liens avec la scène artistique internationale et avec la France.

Il s'agit en effet d'une période où on travaille avec insistance à établir des liens avec la France : c'est d'ailleurs à ce moment-là que la Délégation du Québec est créée. Établir des liens avec la France permettait, d'une part, de défendre les valeurs de modernité en inscrivant l'art québécois sur la scène de l'art contemporain européen, et de rompre avec le nationalisme traditionnel ${ }^{24}$. D'autre part, il n'était pas question de renier pour autant la nécessaire affirmation de la culture nationale. La France présentait à la fois un modèle culturel dont le caractère moderne et francophone rencontrait les objectifs culturels et politiques du gouvernement du Québec au début des années 60. On peut aussi croire que la France avait intérêt à établir des liens avec le Québec. Son hégémonie culturelle avait été mise en question après la Deuxième Guerre mondiale, et c'était New York qui était devenue le centre international de l'art contemporain.

Dès la création du ministère des Affaires culturelles, des contacts établis entre la France et le Québec ont entraîné une série d'événements artistiques ${ }^{25}$. Le premier de ces événements, concernant les arts visuels, fut une exposition intitulée "L'Art au Canada" au Musée de Bordeaux ${ }^{26}$, exposition organisée à la suite d'une invitation de Chaban-Delmas à l'occasion du jumelage des villes de Québec et de Bordeaux. Malgré son titre - "L'Art au Canada" - seul le Québec avait été invité à y participer. L'exposition a permis d'illustrer, pour la première fois sur la scène européenne, l'histoire de l'art québécois, à partir des oeuvres de peintres traditionnels comme Suzor-Côté, Clarence Gagnon, etc., et de quelques

\footnotetext{
G. Frégault, op. cit.

Il est peut-être utile de rappeler ici que le ministère équivalent à celui de Georges-Émile Lapalme était détenu en France par André Malraux qui était venu au Québec en 1963 en réponse à une invitation du ministre québécois. "Sa présence parmi nous, écrit-il, nous consacrait ostensiblement une priorité et nous accordait une place quelque part sur l'échiquier de ce que l'on commençait à appeler la francophonie" (Georges-Émile Lapalme, op. cit., p. 241).

25 Par exemple, La Revue française a lancé un numéro spécial sur le Québec, contenant un article de Guy Viau intitulé "Peintres au Québec".

26 "L'Art au Canada", à Bordeaux - 11 mai - 31 juillet 1962. Voir Georges-Émile Lapalme, op, cit., p. 114.
} 
oeuvres des premiers peintres de la modernité comme Borduas, Riopelle et Pellan. Cette exposition a servi à consolider des liens culturels et politiques entre la France et le Québec ; elle a aussi servi à faire reconnaître le caractère spécifique du Québec sur la scène internationale.

Dans ce mouvement de consolidation des liens entre la France et le Québec, la création de la Délégation du Québec à Paris en 1961 a été un moment important ; Georges-Émile Lapalme nous dit qu'elle a été créée en réponse à une invitation de Malraux. Le premier attaché culturel de la délégation fut Robert Elie, dont les idées sur la modernité en art sont connues. Il a su utiliser un réseau de relations personnelles entre des critiques d'art français et des artistes québécois vivant en France depuis plusieurs années pour réaliser une diffusion européenne de l'art québécois ${ }^{27}$. Un autre événement artistique important, à peu près contemporain de l'exposition de Bordeaux, est la participation de peintres du Québec au Festival de Spolete à l'été 1962. Dans le cadre de ce festival, la Délégation du Québec, aidée de représentants des milieux artistiques québécois et européen, avait organisé une exposition intitulée "La peinture canadienne moderne : 25 ans de peinture canadienne-française" 28 . C'était la première fois qu'était présentée sur la scène européenne une exposition consacrée exclusivement à l'art contemporain québécois, regroupant les peintres des mouvements automatiste, post-automatiste, et plasticien ${ }^{29}$. La peinture gestuelle dominait dans l'exposition. La critique d'art a utilisé l'expression École de Montréal ${ }^{30}$ pour désigner ce mouvement abstrait de la peinture québécoise. Cette expression avait déjà été utilisée par un critique français, Jean Cathelin ${ }^{31}$, qui avait déclaré que l'École de Montréal existait en faisant valoir les sources françaises de la peinture québécoise moderne et en affirmant qu'il existait des affinités entre les peintres abstraits de Montréal et ceux

27 La Galerie Nahmer a fait une exposition intitulée "La Nouvelle École de Montréal" qui regroupait des oeuvres choisies par le critique d'art français Jean Cathelin. L'exposition a été commenté par les revues françaises Cimaise et Aujourd'hui, art et architecture. Cette exposition avait été patronnée par Robert Élie.

28 Festival de Spolete: "La Peinture canadienne moderne : 25 ans de peinture canadiennefrançaise" - 26 juin - 23 août 1962. Parmi les intervenants qui se sont mobilisés pour l'organisation de l'exposition, il y eut Charles Delloye, critique d'art français et collectionneur d'oeuvres de peintres québécois, et M. Sandberg, alors directeur du Stedejik Museum à Amsterdam.

29 "Les pionniers : Pellan, Borduas; le mouvement automatiste : Riopelle, Mousseau, Barbeau, Leduc, Gauvreau, Ferron, Bellefleur ; le post-automatiste : Blair, Comtois, Letendre, Ewen, Boudreau, Champeau, Lise Gervais ; le mouvement plasticien: Molinari, Tousignant, Goguen, Juneau, Toupin ; la tendance surréalisante: Dumouchel, Tremblay. Giguère ; nouvelles recherches : Marcelle Maltais, E. Alleyn, C. Gagnon, J. Lefébure, B. Vanier, R. Arsenault. (Catalogue de l'exposition, "La Peinture canadienne moderne : 25 ans de peinture canadienne-française").

30 Paquerette Villeneuve, "Spoleto, Définitive entrée en scène de la peinture canadienne en Europe", Aujourd'hui, art et architecture, septembre 1962, no 38, p. 46.

31 Jean Cathelin, "L'École de Montréal". Vie des Arts, no 23, été 1961, pp. 14-20. 
de Paris réunis sous l'appellation École de Paris. Par exemple, Jean Cathelin écrit qu'à l'origine de la peinture québécoise moderne, il y a Pellan qui a séjourné à Paris et y a assimilé le modèle français de la culture : "Il retrouve la véritable voie française de l'art (...) il s'intègre parfaitement à la compréhension nouvelle de l'univers pictural qu'impose l'École de Paris" qu'il canadianise", écrit-il ${ }^{32}$.

L'exposition de Spolete, qui était subventionnée par le ministère des Affaires culturelles, a suscité beaucoup d'espoirs dans le milieu artistique québécois qui voyait enfin une possibilité de diffuser l'art québécois sur la scène internationale. Georges-Émile Lapalme lui-même a cru que cette exposition serait le point de départ de la diffusion internationale de l'art québécois: "l'État y était: nos peintres s'y trouvaient, écrit-il, une politique se dessinait en bifurquant vers quelques chose de nouveau, de différent ${ }^{33}$." Mais il n'y eut pas de suite. L'État n'a pas subventionné à court terme d'autres activités visant à mettre sur pied un réseau international de diffusion de l'art québécois. Ce problème de diffusion est encore d'actualité.

Que retenir de ces premiers moments de l'intervention de l'État québécois dans la vie artistique ? Georges-Émile Lapalme a reconnu le rôle initiateur tenu par les artistes durant les années 40 et 50 . Il a cherché à intégrer l'activité artistique dans le projet de société moderne qui était celui du Québec au début des années 60. On peut aujourd'hui juger du caractère utopique de cette intention, étant donné que l'activité artistique n'est pas encore considérée par l'appareil politique comme une activité d'intérêt public. Je pense que le dernier budget du gouvernement Bourassa vient de nous en donner encore une autre preuve.

Fin du texte.

Ibid., $p .16$.

Lapalme, Le Paradis du pouvoir, p. 161. 